\title{
PIV measurements in a turbulent boundary layer overlying a spanwise heterogeneous roughness
}

\author{
R. Yao ${ }^{1}$, K. T. Christensen ${ }^{1,2}$ \\ ${ }^{1}$ University of Notre Dame, Department of Aerospace and Mechanical Engineering, Notre Dame, IN, \\ 46556, USA \\ 2 Illinois Institute of Technology, Department of Mechanical, Materials and Aerospace Engineering \& \\ Civil, Architectural and Environmental Engineering, Chicago, IL, 60616, USA
}

\section{Introduction}

In nature and engineering applications, wall-bounded flow often encounter a heterogeneous surface condition, such as the atmosphere boundary layer at the urban boundary and flow over riveted aircraft surfaces. In a particular scenario, when the surface heterogeneity is predominantly in the spanwise direction of the flow, this roughness heterogeneity can generate secondary flow in cross flow plane which is very different from smooth-wall or homogeneous rough-wall boundary layers.
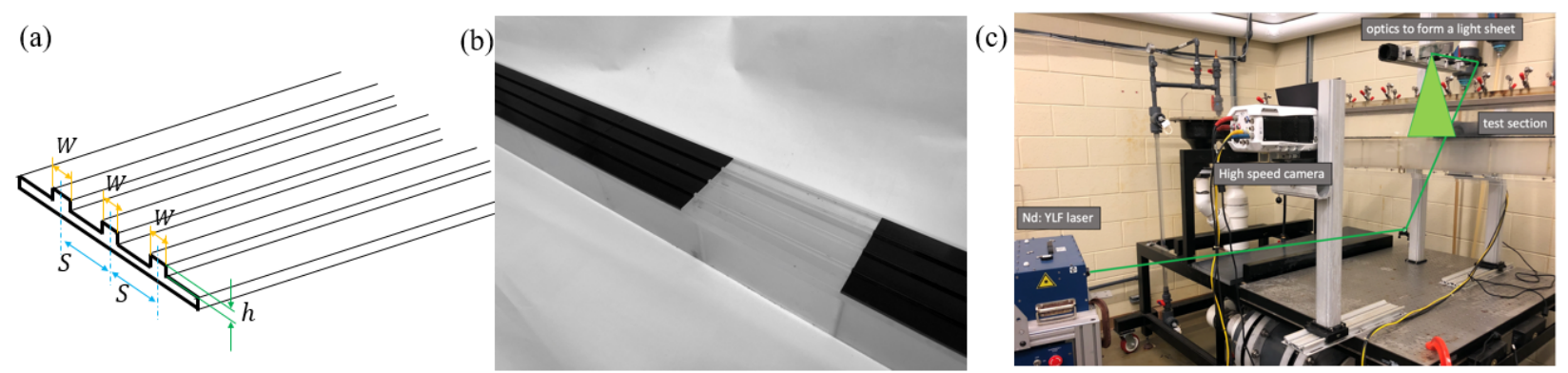

Figure 1: (a) Important parameters of the ridge-type roughness: $h$, ridge height; $S$, the spacing of the ridges; $W$, ridge width. (b) Surface models used in these experiments (the RI of the clear one is matched with that of the working fluid during experiments). (c) Planar PIV setup for streamwise-wall-normal measurements.

A number of experimental (Barros and Christensen, 2014, Vanderwel and Ganapathisubramani, 2015, Zampiron et al. 2020) and numerical (Anderson et al., 2015; Hwang and Lee, 2018; Castro et al., 2021) studies have shown features associated with the secondary flow including: streamwise oriented alternating lowand high-momentum pathways (LMP, HMP); downwash and upwash flow at HMP and LMP, respectively; counter-rotating streamwise vortices formed across an LMP and HMP. The secondary flow mechanism is explained based on Prandtl's secondary flow of the second kind according to Anderson et al. (2015). With this current understanding, this work uses PIV to document the spatio-temporal information of this special flow in $x-y$ and $y-z$ planes over a ridged spanwise heterogeneous rough surface. The results present here mainly focus on the mean and turbulence statistics measured in a flow facility at moderate Reynolds number.

\section{Methods}

The roughness models used in the current experiments are derived from an idealized ridge-type roughness, wherein the roughness topography is constructed from equally spaced bars of square cross-section (Fig 1 a). In the design of the roughness model, the size and spacing of the ridge elements were selected to ensure the generation of strong secondary flows for ridged roughness (Vanderwel and Ganapathisubramani, 2015, Medjnoun et al., 2018). PIV measurements are performed in a refractive-index-matched (RIM) flow facility (Fig. 17) wherein the refractive index of the working fluid (aqueous $\mathrm{NaI}$ ) matches that of solid models of the roughness so that reflections and aberrations in the PIV illumination and imaging are minimized and flow in the immediate vicinity of the roughness can be interrogated by PIV. Figure 1 (b) shows the roughness tiles for RIM and the overall roughness arrangement in the test section. 
(a)

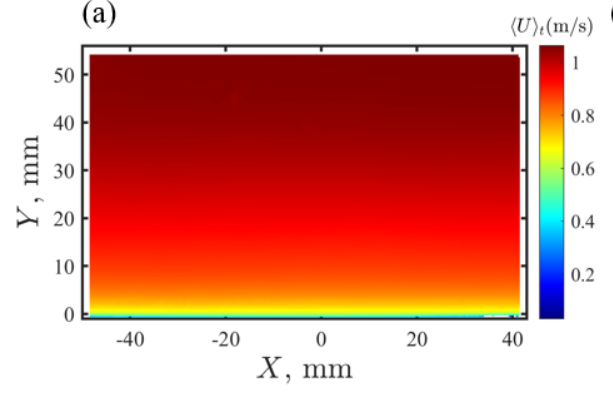

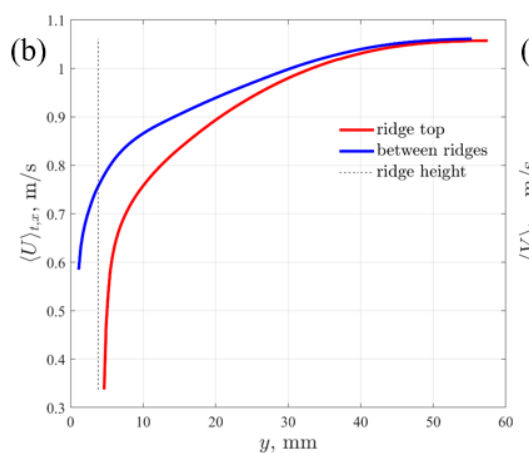

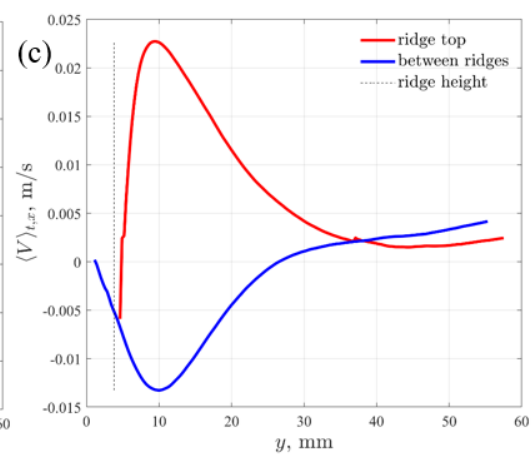

Figure 2: (a) Ensemble-averaged streamwise velocity field between at the center between spanwiseseparated ridges. $(b, c)$ Ensemble and streamwise-averaged streamwise and wall-normal velocity profiles positioned on top of a ridge and between two ridges.

\section{Results}

Initial results showing the time-averaged and time and streamwise-averaged velocities from $x-y$ plane PIV measurements are shown in Fig. 2. Figure 2 a shows the time-averaged streamwise component velocity in the position between two ridges from wall to the free stream. In this case the data below $y=3.8 \mathrm{~mm}$ is behind the ridges and therefore demonstrates the applicability of RIM in this case. Figure $2 \mathrm{a}$ also verifies the streamwise homogeneity of the mean flow. The ensemble- and streamwise-averaged velocity profiles are calculated and shown in Figs. 2b,c. The profiles confirm that the HMP and downwash region is located in between the ridges while the LMP and upwash is observed above the ridges which is consistent with observations in the literature (Vanderwel and Ganapathisubramani, 2015; Medjnoun et al., 2018; Castro et al., 2021). The ongoing progress of this work includes: stereo-PIV measurements in the cross-plane, frequency analysis, and quantification of inner-outer interactions in this flow scenario. Follow-on research will focus on aligning these same ridges obliquely to the mean flow to understand the persistence of these secondary flows relative to the spanwise heterogeneity of the topography.

\section{References}

Anderson W, Barros JM, Christensen KT, and Awasthi A (2015) Numerical and experimental study of mechanisms responsible for turbulent secondary flows in boundary layer flows over spanwise heterogeneous roughness. Journal of Fluid Mechanics 768:316-347

Barros JM and Christensen KT (2014) Observations of turbulent secondary flows in a rough-wall boundary layer. Journal of Fluid Mechanics 748

Castro IP, Kim J, Stroh A, and Lim HC (2021) Channel flow with large longitudinal ribs. Journal of Fluid Mechanics 915

Hwang HG and Lee JH (2018) Secondary flows in turbulent boundary layers over longitudinal surface roughness. Physical Review Fluids 3:014608

Medjnoun T, Vanderwel C, and Ganapathisubramani B (2018) Characteristics of turbulent boundary layers over smooth surfaces with spanwise heterogeneities. Journal of Fluid Mechanics 838:516-543

Vanderwel C and Ganapathisubramani B (2015) Effects of spanwise spacing on large-scale secondary flows in rough-wall turbulent boundary layers. Journal of Fluid Mechanics 774

Zampiron A, Cameron S, and Nikora V (2020) Secondary currents and very-large-scale motions in openchannel flow over streamwise ridges. Journal of Fluid Mechanics 887 